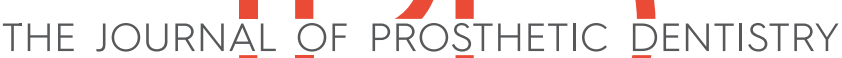

RESEARCH AND EDUCATION

\section{Proinflammatory cytokine production from NOK-SI keratinocytes after exposure to denture adhesives}

\author{
Andressa Rosa Perin Leite, DDS, MSc, ${ }^{a}$ Norberto Martins de Oliveira Júnior, DDS, MSc, ${ }^{b}$ \\ Danny Omar Mendoza Marin, DDS, MSc, ${ }^{c}$ Marco Antonio Compagnoni, DDS, PhD, ${ }^{d}$ and \\ Ana Carolina Pero, DDS, $\mathrm{PhD}^{\mathrm{e}}$
}

Denture adhesives are commercially available products and have been used regularly by millions of denture wearers since the late 18th century. They are formulated from a mixture of short- and long-acting synthetic polymers that hydrate and increase in volume to fill voids between the denture and mucosal tissues. They also increase adhesive and/or cohesive properties and the viscosity of the saliva. ${ }^{1-4}$ When used properly, these products can improve denture retention and stability, decrease occlusion and disocclusion time in complete dentures, ${ }^{5}$ and increase the masticatory efficacy of well-made complete dentures. Especially if the denture adhesive has a copolymer of vinyl methyl ether and maleic anhydride in its composition, ${ }^{6}$ they may also improve denture wearers' perceptions of quality of life. ${ }^{7}$

Denture adhesives do not significantly alter the biofilm formation over a period of 14 days of continuous use and possess antifungal properties. ${ }^{8,9}$ However, denture adhesives may inactivate the activity of antimicrobial peptides and antimicrobial lipids, suggesting

\begin{abstract}
Statement of problem. Although the cytotoxicity of denture adhesives has been widely assessed, data on the release of proinflammatory cytokines from oral epithelial cells are still scarce.

Purpose. The purpose of this in vitro study was to evaluate the proinflammatory cytokines interleukin (IL) $1 \beta$, IL-6, and tumor necrosis factor $\alpha$ (TNF- $\alpha$ ) released from human oral keratinocytes after exposure to denture adhesives.

Material and methods. Eluates of the Ultra Corega cream, Corega powder Fixador Ultra, and Corega strip denture adhesives were created at $1 \% \mathrm{wt} / \mathrm{vol}$ after 24 hours of exposure in a Dulbecco modified Eagle medium culture. The release of IL-1 $\beta, \mathrm{IL}-6$, and TNF- $\alpha$ was measured using a sandwich enzyme-linked immunosorbent assay after the oral epithelial cells (NOK-SI) had been exposed to the eluates for 3,6 , and 24 hours $(n=6)$.
\end{abstract}

Results. IL-1 $\beta$ release from the strip was higher at 3 hours compared with the control (Bonferroni post hoc test, $P=.016)$. IL- 6 releases from the cream and powder denture adhesives at 6 and 24 hours were higher than for the controls (Bonferroni post hoc test, $P<.05$ ). TNF- $\alpha$ release was not detectable in some groups and periods.

Conclusions. In long periods, denture adhesive strips do not induce any change on cytokine release from human oral keratinocytes. (J Prosthet Dent 2018;119:404-8)

that components in adhesives may inactivate local innate immune factors in the oral cavity, possibly leading to the growth of Candida albicans and inducing denture stomatitis. ${ }^{10}$ In addition, the chronic and excessive use of zinc-containing denture adhesives has been recognized as a potential cause of copper deficiency and hyperzincemia with resultant neurologic symptoms.

The difficulty of removing denture adhesives is a common problem, and it seems to be more effective to

Supported by the Brazilian National Research Council (CNPQ; grant 158672/2013-5).

a Doctoral student, Department of Dental Materials and Prosthodontics, Araraquara Dental School, São Paulo State University (UNESP), Araraquara, Brazil.

${ }^{b}$ Doctoral student, Department of Dental Materials and Prosthodontics, Araraquara Dental School, São Paulo State University (UNESP), Araraquara, Brazil.

${ }^{\mathrm{c} D o c t o r a l}$ student, Department of Dental Materials and Prosthodontics, Araraquara Dental School, São Paulo State University (UNESP), Araraquara, Brazil.

${ }^{d}$ Professor, Department of Dental Materials and Prosthodontics, Araraquara Dental School, São Paulo State University (UNESP), Araraquara, Brazil.

eAssistant Professor, Department of Dental Materials and Prosthodontics, Araraquara Dental School, São Paulo State University (UNESP), Araraquara, Brazil. 


\section{Clinical Implications}

Denture adhesive strips do not induce change in cytokine release from keratinocytes in long periods, which may make it more useful than the other tested denture adhesives. The cream and powder denture adhesive increased the release of the proinflammatory cytokines, which may make it somewhat less useful than the strips. The use of cream and powder adhesives for shorter periods would be safer.

brush the dentures with coconut soap, dentifrice, or water combined with immersion in sodium perborate solution. ${ }^{11}$ Antifungal agents, especially chlorhexidine dihydrochloride and fluconazole, could be candidates for inclusion in denture adhesive formulations and used as prescribed topical treatments for individuals with denture stomatitis. ${ }^{10}$

The ideal denture adhesive should be nontoxic, nonirritating, and biocompatible with the oral mucosa. ${ }^{12}$ Cytotoxic ingredients in these products may be released into the oral cavity. ${ }^{13}$ Additionally, previous studies have identified their cytotoxic potential against cell lines in vitro, ${ }^{13-18}$ and some commercial adhesives have been shown to release formaldehyde. ${ }^{19,20}$ These studies have examined the cytotoxicity of denture adhesives to human oral keratinocytes, ${ }^{14}$ human oral fibroblasts, ${ }^{14,16-18}$ and a mouse fibroblast cell line ${ }^{13-15,18}$ (L929) by using a wide variety of assay techniques, and varying levels of cytotoxicity have been reported.

However, studies considering cytotoxicity levels in epithelial cell lines collected from gingival tissue ${ }^{14}$ are rare, and the authors are unaware of studies evaluating the release of proinflammatory cytokines and the role of denture adhesives in inflammation process. Changes in levels of these cytokines may serve as sensitive indicators of adverse biologic effects of denture adhesives. ${ }^{21}$ Therefore, the purpose of this study was to assess the effect of denture adhesives on the release of proinflammatory cytokines (interleukin [IL] $1 \beta$, IL-6, and tumor necrosis factor alpha [TNF- $\alpha]$ ) derived from spontaneously immortalized normal oral keratinocytes (NOK-SI). The null hypothesis was that neither the type of denture adhesive nor the exposure period would influence the release of cytokines.

\section{MATERIAL AND METHODS}

NOK-SI ${ }^{22}$ were cultured in Dulbecco modified Eagle medium (DMEM; Gibco) supplemented with 10\% fetal bovine serum, $100 \mathrm{IU} / \mathrm{mL}$ penicillin, $100 \mu \mathrm{g} / \mathrm{mL}$ streptomycin, and $2 \mathrm{mmol} / \mathrm{L}$ glutamine (penicillinstreptomycin-glutamine 100x; Gibco). The cultures were kept in $75 \mathrm{~cm}^{2}$ culture flasks at $37^{\circ} \mathrm{C}$ in a humidified $5 \%$ $\mathrm{CO}_{2}$ balanced air incubator. After reaching approximately $90 \%$ cell density, the cells were trypsinized, seeded in sterile 6-well plates $\left(3.5 \times 10^{5}\right.$ cells per well), and incubated in an atmosphere of $5 \% \mathrm{CO}_{2}$ and $95 \%$ air at $37^{\circ} \mathrm{C}$ for 24 hours. The cells were used between the fourth and eighth passages in the experiments.

The appropriate amounts of each denture adhesive (Ultra Corega cream, Corega powder Fixador Ultra, and Corega strips; GlaxoSmithKline Brasil Ltda) were added to the supplemented DMEM without serum to yield a $1 \%$ $\mathrm{wt} / \mathrm{vol}$ eluate. The denture adhesives were weighed on a precision scale in a falcon-type tube, and then the DMEM culture medium was added to the tubes with the aid of a serologic pipette and $100 \mathrm{IU} / \mathrm{mL}$ penicillin, 100 $\mu \mathrm{g} / \mathrm{mL}$ streptomycin, and $2 \mathrm{mmol} / \mathrm{L}$ glutamine. The tube was closed, and a sealing film (Parafilm M; SigmaAldrich) was wrapped around the cap. Thus, the DMEM culture medium remained in contact with the denture adhesive for 24 hours. Next, the aqueous extract was centrifuged and filter-sterilized using a $0.22 \mu \mathrm{m}$ filter (syringe filter $0.22 \mu \mathrm{m}$ \#99722; TPP). Higher concentrations of eluates were not tested because they became too viscous to be filter-sterilized. ${ }^{14}$ The extracts were supplemented with $10 \%$ fetal bovine serum and then used in the experiments.

After 24 hours, the culture medium was replaced by $3 \mathrm{~mL}$ of eluates obtained from the denture adhesives or by supplemented DMEM (control group) in duplicate and on 3 different occasions $(n=6)$. After 3, 6, and 24 hours, the supernatant of the cells was collected and stored in a freezer at $-80^{\circ} \mathrm{C}$ until the experiment.

The cell culture supernatant was examined using enzyme-linked immunosorbent assay (ELISA) to quantify any IL-1 $\beta$ (human IL-1 $\beta$ ELISA kit KHC0011; Invitrogen), IL-6 (human IL-6 ELISA kit KHC0061; Invitrogen), or TNF- $\alpha$ (human TNF- $\alpha$ ELISA kit KHC3011; Invitrogen) present, according to the manufacturer's instructions. Briefly, $100 \mu \mathrm{L}$ of standard IL-1 $\beta$, IL-6, or TNF- $\alpha$ or culture supernatants or controls were added to the coated wells to allow the antigen from the specimens to bind to the immobilized (capture) antibody. After washing, a detection antibody was added that bound to the immobilized antigen captured during the first incubation. The excess of detection antibody was removed and streptavidin-horseradish peroxidase was added. After a second incubation and washing to remove the entire unbound enzyme, a stabilized substrate solution was added. The reaction was stopped by the addition of $100 \mu \mathrm{L}$ of stop solution, and the plates were read at $450 \mathrm{~nm}$ in an ELISA microplate reader (VersaMax ELISA microplate reader; Molecular Devices). The cytokine concentrations were determined using interpolation from a standard curve and have been presented as picograms per milliliter $( \pm S D)$ for 
Table 1. Two-way ANOVA for IL-1 $\beta$ released

\begin{tabular}{lccccc}
\hline Source of Variation & SS & df & MS & F & $\boldsymbol{P}$ \\
\hline Groups & 0.069 & 3 & 0.023 & 1.78 & .164 \\
\hline Time & 0.179 & 2 & 0.09 & 6.933 & .002 \\
\hline Groups $\times$ Time & 0.266 & 6 & 0.044 & 3.432 & .007 \\
\hline Error & 0.608 & 47 & 0.013 & & \\
\hline
\end{tabular}

$\mathrm{IL}$, interleukin; SS, sum of squares; MS, mean square. $P<.05$ denotes significant statistical difference.

each of the tested conditions. The ELISA assay was performed using the cell supernatant of these duplicates on 3 occasions ( $n=6$ wells per group).

Statistical analyses were performed using software (SPSS Statistics for Windows, v17.0; SPSS Inc) and 2-way ANOVA followed by the Bonferroni post hoc test in order to identify whether any groups differed significantly in their release of IL-1 $\beta$ or IL-6 $(\alpha=.05)$. A multiple linear regression model using the stepwise method for selection of independent variables was applied to assess the relationship between the release of IL- $1 \beta$ and IL- 6 with the time and groups as predictors $(\alpha=.05)$. Data on the release of TNF- $\alpha$ were analyzed descriptively.

\section{RESULTS}

Within the same period, IL-1 $\beta$ release from strip denture adhesive was higher than the control at 3 hours $(P=.016)$. The extent of IL-1 $\beta$ release in response to the cream denture adhesives at 24 hours of exposure was significantly higher than at 3 hours $(P=.029)$ and in response to the powder denture adhesive was higher at 6 hours $(P=.004)$ and 24 hours $(P=.009)$ than at 3 hours. However, IL-1 $\beta$ releases at 6 hours and 24 hours were similar to those of the controls in the experiments on these 2 adhesives $(P>.05)$ (Table 1, Fig. 1).

Within the same period, IL-6 release was found to be similar among all groups, including the control at 3 hours $(P>.05)$. Higher release of IL-6 was observed in the experiment on the cream and powder denture adhesives at 6 hours ( $P=.001, P=.007$, respectively) and at 24 hours (both $P<.001)$. For all groups, the release of IL-6 increased with time $(P<.05)$ (Table 2, Fig. 2).

After evaluation with a multiple linear regression model, the variable independent time was identified as a significant predictor for IL- $1 \beta$ and IL- 6 . According to the linear regression model, the coefficient of determination $\left(\mathrm{R}^{2}\right)$ showed that $13.3 \%$ of the variation of IL- $1 \beta$ release and $86.6 \%$ of the variation IL- 6 release can be explained by the time. The analysis of ANOVA showed a significant linear relationship between the time variable and IL-1 $\beta$ $(P=.005)$ and IL-6 $(P<.001)$, showing that when the time increases, the values obtained for IL-1 $\beta$ and IL-6 increase.

Data on the TNF- $\alpha$ release obtained from the ELISA microplate reader and plotting the standard curve were

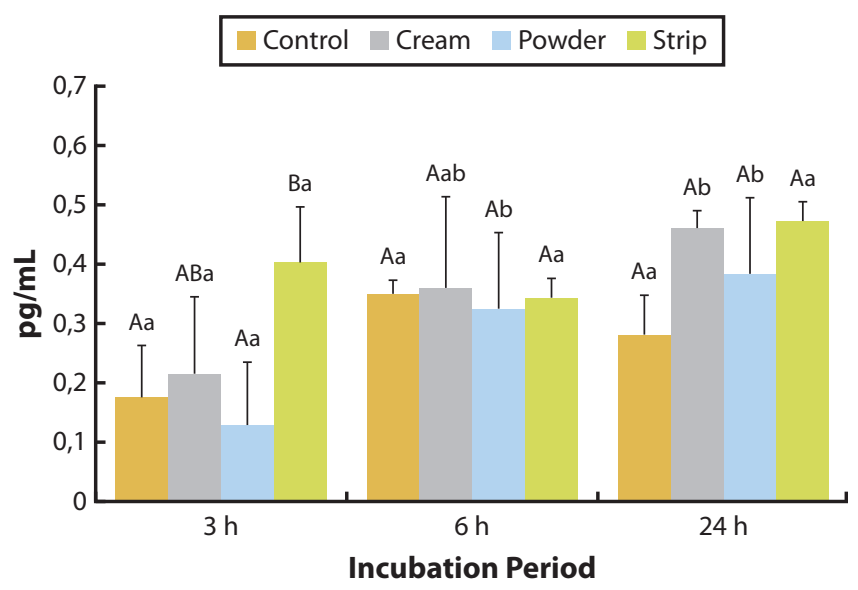

Figure 1. Mean values and SD of interleukin (IL) $1 \beta$ release in each experimental group during incubation periods assessed $(3,6$, and 24 hours). Within same period, identical uppercase letters denote statistical similarity among groups; within same group, lowercase letters denote statistical similarity among times $(P>.05)$.

Table 2. Two-way ANOVA for IL-6 released

\begin{tabular}{lccccc}
\hline Source of Variation & SS & df & MS & F & $\boldsymbol{P}$ \\
\hline Groups & 1307.127 & 3 & 435.709 & 24.842 & $<.001$ \\
\hline Time & 17568.757 & 2 & 8784.379 & 500.834 & $<.001$ \\
\hline Groups $\times$ Time & 358.641 & 6 & 59.773 & 3.408 & .005 \\
\hline Error & 1157.607 & 66 & 17.54 & & \\
\hline
\end{tabular}

$\mathrm{IL}$, interleukin; SS, sum of squares; MS, mean square. $P<.05$ denotes significant statistical difference.

not detectable in some groups and periods (Table 3). Thus, statistical analysis was not performed.

\section{DISCUSSION}

The null hypothesis of this study was rejected because the release of IL- $1 \beta$ and IL- 6 was influenced by the type of denture adhesive and by the exposure time.

IL- $1 \beta$, IL- 6 , and TNF- $\alpha$ are proinflammatory cytokines that appear to play a central role in inflammatory processes. They were measured in this study to investigate a possible link between denture adhesives and any adverse biologic effects of their materials, such as acute toxicity. ${ }^{19,20}$ IL-1 $\beta$ mediates inflammatory response by promoting recruitment of phagocytes, cytokine regulation, and chemokine production. It also promotes bone resorption, cell proliferation, and differentiation in response to inflammatory stimuli. ${ }^{24}$ IL-6 is associated with inflammation and immune response processes, including vascular permeability, osteoclast differentiation, and bone resorption regulation mechanisms; the deregulation of its signaling contributes to the onset and maintenance of several diseases, including rheumatoid arthritis and osteoporosis. ${ }^{25,26} \mathrm{TNF}-\alpha$ is a pleiotropic proinflammatory cytokine involved in systemic and local 


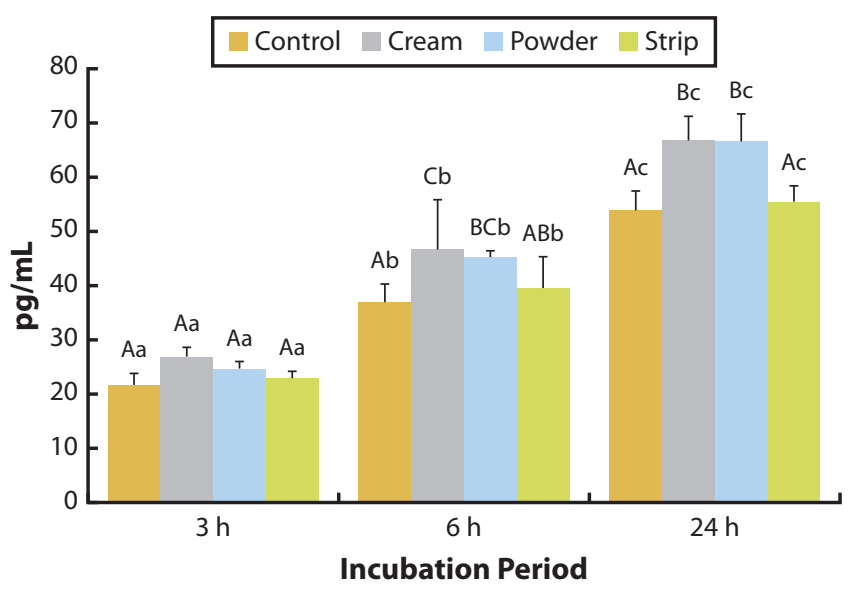

Figure 2. Mean values and SD of interleukin (IL) 6 release in each experimental group during incubation periods assessed $(3,6$, and 24 hours). Within same period, identical uppercase letters denote statistical similarity among groups; within same group, lowercase letters denote statistical similarity among times $(P>.05)$.

inflammation such as the induction of inflammatory mediators and it one of the most important osteoclastactivating cytokines produced during inflammatory processes. $^{27}$

The main findings of this study were an increase in IL-6 production by NOK-SI in the experiments on denture adhesive cream and powder after 6 or 24 hours of exposure. A significant increase of IL-1 $\beta$ release was found in strip denture adhesives at 3 hours. Cytokine release was measured in order to investigate the possible link between denture adhesives and clinical parameters such as inflammation. ${ }^{23}$

The authors are unaware of previous studies that have assessed proinflammatory cytokine release from keratinocytes exposed to denture adhesives. Previous studies have evaluated the influence of denture adhesives and their ingredients using cytotoxicity assays. ${ }^{12,14,17-19}$ The effect of denture adhesives on oral keratinocytes was reported in only one study, which concluded that primary human oral mucosal cells may provide more valuable information in toxicity screenings of denture adhesives. ${ }^{14}$ All of the denture adhesives tested were found to be slightly to moderately cytotoxic to primary keratinocytes but nontoxic to L929 cells. ${ }^{14}$ Other studies have evaluated the effects of denture adhesives on mouse fibroblast cells (L929) and immortalized human gingival fibroblasts. ${ }^{13,15-19}$ Human gingival keratinocytes have been used for cytotoxicity testing because they are in close contact with denture adhesives and consequently with any chemicals leached from these materials in the oral cavity; therefore, their use in experiments is more clinically relevant. ${ }^{14}$

Denture adhesives are typically not diluted before use. In this study, however, the denture adhesives were filter-sterilized to avoid bias and confounding results.
Table 3. Means (SDs) of TNF- $\alpha$ released from NOK-S

\begin{tabular}{lccc}
\hline & \multicolumn{4}{c}{ Time (h) } \\
\cline { 2 - 4 } Group & $\mathbf{3}$ & $\mathbf{6}$ & $\mathbf{2 4}$ \\
\hline Control & ND & ND & ND \\
\hline Cream & ND & ND & ND \\
\hline Powder & ND & $1.124(0.43)$ & $1.473(0.68)$ \\
\hline Strip & ND & ND & $1.633(1.15)$ \\
\hline
\end{tabular}

ND, not detectable. Shown is tumor necrosis factor alpha (TNF- $\alpha$ ) released from oral epithelial cells (NOK-SI) as assessed by enzyme-linked immunosorbent assay after contact with denture adhesive eluates for 3,6 , and 24 hours. Reaction product (pg/mL) measured as optical density at $450 \mathrm{~nm}$.

Adhesives may contain microbial contamination, which may lead to the incorrect release of the inflammation molecules in the culture medium. ${ }^{13,19,28,29}$ Thus, the constituents leached out of the adhesives in this study most likely caused the cytotoxicity. ${ }^{13}$

The release of formaldehyde, a component that displays a high level of cytotoxicity, has previously been demonstrated. ${ }^{19,20}$ This component exhibits a high level of cytotoxicity and is responsible for allergic inflammation in patients wearing dentures. ${ }^{19,21,30}$ Some authors have suggested that the lower cytotoxicity of certain denture adhesives could be associated with the absence of formaldehyde. ${ }^{13,16,17}$ A previous report showed that one denture adhesive includes diazolidinyl urea as an ingredient, which is a formaldehyde releaser; thus, the authors inferred that diazolidinyl urea was the cause of the significantly lower cell viability and increased rates of apoptosis found in their study. ${ }^{17}$ In a study using MTT ((4,5-dimethylthiazol-2-yl)-2,5-diphenyltetrazolium bromide) assay and agar diffusion, $\mathrm{Al}$ et $\mathrm{al}^{13}$ determined that one of the adhesives tested produced severe cytotoxic effects on the L929 cell line. The authors noted that this observation may have been because this was the only adhesive that had maleic acid and methoxyethene in its composition.

Short and intermediate exposure times were used ( 3 and 6 hours) in an attempt to simulate adhesive use among patients who clean their dentures after each meal, whereas the prolonged exposure period ( 24 hours) simulated the difficulty in removing these products because most denture wearers are elderly and may have less motor control. The results of this study indicate that denture adhesive strips may be more favorable to use over long periods. Although in vitro findings cannot be directly extrapolated to clinical situations, oral mucosal cells are protected by mucin and also in part by keratinization; in addition, the cytotoxic effect of the adhesive is likely to be lower. ${ }^{14}$ Furthermore, saliva does not continuously dilute the denture adhesive in the clinical setting. ${ }^{31}$ Further research is necessary to investigate which parameters affect cytokine release from single layer and 3-dimensional cultures of normal cells, and in vivo studies are needed to determine the true behavior of these products in the oral cavity. 


\section{CONCLUSIONS}

On the basis of the findings of this in vitro study, the following conclusions were drawn:

1. Denture adhesive strips did not induce change of cytokine release from human oral keratinocytes after long periods and may be easier to use.

2. The cream and powder denture adhesives can induce cytokine release after long periods.

\section{REFERENCES}

1. Figueiral MH, Fonseca PA, Pereira-Leite C, Scully C. The effect of different adhesive materials on retention of maxillary complete dentures. Int J Prosthodont 2011;24:175-7.

2. Munoz CA, Gendreau L, Shanga G, Magnuszewski T, Fernandez P, Durocher J. A clinical study to evaluate denture adhesive use in well-fitting dentures. J Prosthodont 2012;21:123-9.

3. Polyzois G, Lagouvardos P, Frangou M, Stefaniotis T. Efficacy of denture adhesives in maxillary dentures using gnathodynamometry: a comparative study. Odontology 2011;99:155-61.

4. Polyzois G, Stefaniotis T, Papaparaskevas I, Donta C. Antimicrobial efficacy of denture adhesives on some oral malodor-related microbes. Odontology 2013;101:103-7.

5. Abdelnabi MH, Swelem AA, Al-Dharrab AA. Influence of denture adhesives on occlusion and disocclusion times. J Prosthet Dent 2016;115:306-12.

6. Torres-Sanchez C, Montoya-Salazar V, Torres-Lagares D, Gutierrez-Perez JL, Jimenez-Castellanos E. Comparison of masticatory efficacy among complete denture wearers with two adhesives and dentate individuals: a randomized, crossover, double-blind clinical trial. J Prosthet Dent 2017; 117:614-20.

7. Felton D, Cooper L, Duqum I, Minsley G, Guckes A, Haug S, et al. Evidencebased guidelines for the care and maintenance of complete dentures: a publication of the American College of Prosthodontists. J Am Dent Assoc 2011;142(suppl 1):1S-20S

8. Leite AR, Mendoza-Marin DO, Paleari AG, Rodriguez LS, Roccia AA, Policastro VB, et al. Crossover clinical trial of the influence of the use of adhesive on biofilm formation. J Prosthet Dent 2014;112:349-56.

9. Rajaram A, Manoj SS. Influence of 3 different forms of a commercially available denture adhesive material on the growth of Candida species: an in vitro study. J Prosthet Dent 2017;118:379-85.

10. Garaicoa JL, Fischer CL, Bates AM, Holloway J, Avila-Ortiz G, Guthmiller JM, et al. Promise of combining antifungal agents in denture adhesives to fight Candida species infections. J Prosthodont 21 Nov 2016. doi 10.1111/jopr.12565. [Epub ahead of print].

11. Nunes E, Policastro VB, Scavassin PM, Leite AR, Mendoza Marin DO, Giro G, et al. Crossover clinical trial of different methods of removing a denture adhesive and the influence on the oral microbiota. J Prosthet Dent 2016:115:462-8

12. Adisman IK. The use of denture adhesives as an aid to denture treatment. J Prosthet Dent 1989:62:711-5.

13. Al RH, Dahl JE, Morisbak E, Polyzois GL. Irritation and cytotoxic potential of denture adhesives. Gerodontology 2005;22:177-83.

14. Chen F, Wu T, Cheng X. Cytotoxic effects of denture adhesives on primary human oral keratinocytes, fibroblasts and permanent L929 cell lines. Gerodontology 2014;31:4-10.

15. de Gomes PS, Figueiral MH, Fernandes MH, Scully C. Cytotoxicity of denture adhesives. Clin Oral Investig 2011;15:885-93
16. DeVengencie J, Ng MC, Ford P, Iacopino AM. In vitro evaluation of denture adhesives: possible efficacy of complex carbohydrates. Int J Prosthodont 1997; 10:61-72.

17. Lee Y, Ahn JS, Yi YA, Chung SH, Yoo YJ, Ju SW, et al. Cytotoxicity of four denture adhesives on human gingival fibroblast cells. Acta Odontol Scand 2015;73:87-92.

18. Zhao K, Cheng XR, Chao YL, Li ZA, Han GL. Laboratory evaluation of a new denture adhesive. Dent Mater 2004;20:419-24.

19. Ekstrand K, Hensten-Pettersen A, Kullmann A. Denture adhesives: cytotoxicity, microbial contamination, and formaldehyde content. J Prosthet Dent 1993;69:314-7.

20. Ellis B, Al-Nakash S, Lamb DJ. The composition and rheology of denture adhesives. J Dent 1980;8:109-18.

21. Schmalz G, Schweik1 H, Hiller KA. Release of prostaglandin E2, IL-6 and IL-8 from human oral epithelial culture models after exposure to compounds of dental materials. Eur J Oral Sci 2000;108:442-8.

22. Castilho RM, Squarize CH, Leelahavanichkul K, Zheng Y, Bugge T, Gutkind JS. Rac1 is required for epithelial stem cell function during dermal and oral mucosal wound healing but not for tissue homeostasis in mice. PLoS One 2010;5:e10503.

23. Moharamzadeh K, Van Noort R, Brook IM, Scutt AM. Cytotoxicity of resin monomers on human gingival fibroblasts and HaCaT keratinocytes. Dent Mater 2007;23:40-4

24. Gomes FI, Aragao MG, Barbosa FC, Bezerra MM, de Paulo Teixeira Pinto V, Chaves HV. Inflammatory cytokines interleukin-1beta and tumour necrosis factor-alpha-novel biomarkers for the detection of periodontal diseases: a literature review. J Oral Maxillofac Res 2016;30:e2.

25. Ghandadi M, Sahebkar A. Curcumin: an effective inhibitor of interleukin-6. Curr Pharm Des 2017:23:921-31.

26. Heinrich PC, Behrmann I, Haan S, Hermanns HM, Muller-Newen G, Schaper F. Principles of interleukin (IL)-6-type cytokine signalling and its regulation. Biochem J 2003;374:1-20.

27. Kwan Tat S, Padrines M, Theoleyre S, Heymann D, Fortun Y. IL-6, RANKL, TNF-alpha/IL-1: interrelations in bone resorption pathophysiology. Cytokine Growth Factor Rev 2004:15:49-60.

28. Gates WD, Goldschmidt M, Kramer D. Microbial contamination in four commercially available denture adhesives. J Prosthet Dent 1994;71 154-8.

29. Makihira S, Nikawa H, Satonobu SV, Jin C, Hamada T. Growth of Candida species on commercial denture adhesives in vitro. Int J Prosthodont 2001;14: 48-52.

30. Kallus T. Enhanced tissue response to denture base polymers in formaldehyde-sensitized guinea pigs. J Prosthet Dent 1984;52:292-9.

31. Darwish M, Nassani MZ. Evaluation of the effect of denture adhesives on surface roughness of two chemically different denture base resins. Eur J Dent 2016;10:321-6.

\section{Corresponding author:}

Dr Ana Carolina Pero

Araraquara Dental School

Rua Humaitá 1680

14801-903 Araraquara, São Paulo

BRAZIL

Email: anacarolpero@foar.unesp.br

\section{Acknowledgments}

The authors thank Dr Carlos Rossa Júnior, Dental School of the São Paulo State University (UNESP), Araraquara Campus and Professor Rogério M. Castilho, University of Michigan, School of Dentistry, for donating the NOK-SI; and Kahena Rodrigues Soldati, Dental School of the São Paulo State University (UNESP), Araraquara Campus.

Copyright () 2017 by the Editorial Council for The Journal of Prosthetic Dentistry. 